Nature Biotech (2008) 26: 6: 653-654

\title{
Stable transgenes bear fruit
}

Ajay Kohli ${ }^{1}$ and Paul Christou ${ }^{2 *}$

1. Institute for Research on Environment \& Sustainability (IRES), Devonshire Building. University of Newcastle upon Tyne, Newcastle - NE1 7RU, UK;

2. ICREA, Universitat de Lleida, PVCF, Av Alcalde Rovira Roure, 191, E-25198, Lleida, Spain

* Author for correspondence (christou@pvcf.udl.es)

Since the first transgenic plants were generated in the 1980 s, over 150 plant species have been transformed for countless different traits, and today nearly 300 million acres of agricultural land are planted with GM crops. Despite the strong science base and the healthy year-on-year growth of GM agriculture, there is surprisingly little diversity - most of the land used for GM is planted with one of four crops (corn, soybean, cotton or canola) and with few exceptions, the plants are transgenic for just two traits: herbicide tolerance and/or pest resistance. This strange juxtaposition of incredible diversity in the laboratory and virtual homogeny in the field reflects in part the cumbersome and internationally disharmonious regulatory environment, which includes risk-assessments based on difficult-to-verify concepts such as transgene stability and the pleiotropic effects of transgene integration. This places an overwhelming burden of proof on developers and leads to long development times ${ }^{1}$.

Bucking the trend somewhat is the SUNUP variety of papaya, a transgenic cultivar carrying a gene for resistance against Papaya ring spot virus (PRSV). The rapid deployment of SUNUP has enhanced the export-dependent economy of a region of Hawaii that was otherwise destined to suffer regular PRSV epidemics. A recent article in Nature by Ming and colleagues ${ }^{2}$ reports the complete genome sequence of the SUNUP papaya variety, the third complete genome sequence of a multicellular plant to be published (after Arabidopsis and rice) and the first ever genome sequence of a transgenic organism. The significance of this sequencing project from a GM perspective is that it supports the biosafety of GM crops by providing definitive molecular evidence against in situ transgene rearrangements, one of the main suspected causes of 'transgene instability'. Although there was no comparative sequencing over multiple generations, the fact that the transgene remains structurally 
and functionally intact in this distant descendent of the original transgenic event is convincing proof that transgenes generally become fixed aspects of the plant genome, and can achieve a consistent and predictable expression level from generation to generation.

A whole genome shotgun (WGS) approach facilitated the sequencing of over $90 \%$ of the euchromatic papaya genome, including $92.1 \%$ of previously identified ESTs, $92.4 \%$ of known genetic markers and the genomic sequences surrounding the integrated transgene DNA. These flanking sequences appear very similar to those around 'natural' DNA integration events, such as the occasional integration of chloroplast DNA fragments into the papaya genome, as reported in the same publication $^{2}$, and tobacco genome, as reported previously ${ }^{3}$. This supports research on both direct DNA transfer (mostly particle bombardment) and Agrobacteriummediated transformation, which has shown that transgene integration is the consequence of a natural process carried out by enzymes involved in DNA break and repair ${ }^{4}$.

The SUNUP genome sequence lends support to many aspects of the current model for transgene integration in plants (Figure 1) and suggests identical methods are involved in artificial and 'natural' DNA integration events. These include the tendency for exogenous DNA sequences to undergo recombination, rearrangement and truncation prior to or during integration (but rarely after integration), the tendency for exogenous DNA to integrate at AT-rich sites resembling topisomerase I recognition sequences, and the tendency for junction regions to show evidence of microhomology and nontemplated DNA synthesis (filler DNA) ${ }^{4}$. All these processes occur regardless of the transformation method because they rely on enzymes endogenous to the nucleus of the host cell.

The initial stages of transgene integration are characterized by complex rearrangements of input sequences resulting from a combination of homologydependent and homology-independent processes stimulated by the presence of a high concentration of free DNA ends (Figure 1a). This often results in the formation of transgene concatemers that may comprise any number of genes, from fewer than 10 to more than 100, some of which are complete and some truncated (Figure 1b). These structures compete with individual genes for integration sites, which are thought to reflect the positions of naturally occurring DNA nicks and breaks ${ }^{5}$. DNA repair 
complexes in the vicinity of such breaks are thought to incorporate exogenous DNA into the repair, generating complex structures that need to be resolved before the DNA duplex can be sealed. The frequent occurrence of topoisomerase sites near to both integrated transgenes and natural integration sites suggests that this enzyme plays an important role in the resolution process (Figure 1c). The fact that most transgenic plants have a single integration site suggests the efficiency of the integration process is low, but the presence of transgene arrays interspersed with genomic DNA in some transgenic plants indicates that the integration process might stimulate further integration events nearby, perhaps through the recruitment of DNA repair complexes (Figure 1d).

Interestingly, the SUNUP genome contains truncated versions of the tet A and nptII marker genes, along with significant amounts of vector backbone $\mathrm{DNA}^{2}$. Such events are commonly seen with both direct DNA transfer and Agrobacterium-mediated transformation ${ }^{4}$ even though in the latter case only the T-DNA is meant to be transferred. Vector DNA integrated during Agrobacterium-mediated transformation can either be linked to the T-DNA, indicating co-linear transfer, or can integrate independently, suggesting that aberrant vector DNA transferred to the plant cell in a co-linear fashion can be fragmented and then integrated like any other exogenous sequence. Alternatively vector DNA can be integrated through non-colinear transfer through juxtaposition of segments adjacent to the T-DNA border after integration (personal communication Dr Hong Yan, TLL, Singapore).

The presence of topoisomerase sites at transgene/genomic junctions following direct DNA transfer ${ }^{5}$ and Agrobacterium-mediated transformation ${ }^{6}$ as well as flanking integrated chloroplast DNA sequences in the papaya genome ${ }^{2}$ may even imply that nuclease activity trims back linear DNA until such sites are exposed, and the attachment of topoisomerase initiates the joining process. Although the SUNUP papaya variety demonstrates transgene stability over several generations, the fact remains that some non-essential sequences are present in the genome. Whereas counter-selective markers can limit the co-transfer of vector sequences by Agrobacterium-mediated transformation, unlinked fragments of the vector can still integrate. With direct transfer, however, vector/marker integration can be completely eliminated through the use of linear cassettes that contain only the promoter, open reading frame and terminator ${ }^{7}$. 
No endogenous genes were interrupted by the exogenous DNA introduced into the SUNUP papaya genome so the transgenic plants are functionally similar to their nontransformed counterparts in all but the virus-resistance phenotype. However, comparative sequencing of the equivalent genomic region in non-transformed papaya has not been reported so it is not clear whether endogenous DNA has been deleted at the integration site or whether any filler DNA has been added at the junctions. It would be very useful to carry out additional sequencing experiments, which could be completed rapidly by using the polymerase chain reaction to amplify across the unused integration site in non-transformed plants using primers flanking the transgene in the SUNUP variety.

Despite the stability of SUNUP papaya, it is clear that current strategies for gene transfer to plants still rely on chance events and the selection of very rare transformants with appropriate properties from a large number of primary events. An alternative transformation process that may become more applicable in the future is transgene integration mediated by homologous recombination, which would allow transgenes to be targeted to specific genomic loci known to be favorable for stable, high-level expression. Although achieved at a low efficiency in model species, the recent reports of homologous recombination in rice $^{8}$ and maize ${ }^{9}$ shows how it could be applied to food crops in the future. Another strategy that may facilitate predictable transgene expression is the control of gene expression through transcription factories. This has been elegantly demonstrated in experiments showing the role of interchromatin interactions between two genes on two different chromosomes that come together at a single focus for transcription, facilitated by the estrogen receptor ${ }^{10}$. Designing constructs that include sequences responsive to transcription factories may ensure stable expression in future transgenic plants. With such advanced technologies at our disposal, one day the creation of functional transgenic plants may cease to be a numbers game and become a more precise science.

\section{References:}

1. Smyth S and McHughen A (2008) Regulating innovative crop technologies in Canada: the case of regulating genetically modified crops PBJ 6: 213-225 
2. Ming R et al. (2008) the draft genome of the transgenic tropical fruit tree papaya (Carica papaya Linnaeus) Nature. 452:991-997

3. Huang CY, Ayliffe MA, Timmis JN (2004) Simple and complex nuclear loci created by newly transferred chloroplast DNA in tobacco. Proc. Natl. Acad. Sciences USA 101: 9710-9715

4. Kohli A et al. (2003) Transgene integration, organization and interaction in plants. Plant Mol Biol 52:247-258

5. Kohli A et al. (1998) Transgene organization in rice engineered through direct DNA transfer supports a two-phase integration mechanism mediated by the establishment of integration hot spots. Proc. Natl. Acad. Sciences USA 95: 7203-7208

6. Makarevitch I, Somers DA (2006) Association of Arabidopsis topoisomerase IIA cleavage sites with functional genomic elements and T-DNA loci. Plant Journal. 48: 697-709

7. Fu XD et al (2000) Linear transgene constructs lacking vector backbone sequences generate low-copy-number transgenic plants with simple integration patterns. Transgenic Research 9: 11-19

8. Terada R et al. (2002) Efficient gene targeting by homologous recombination in rice. Nature Biotechnology 20, 1030 - 1034

9. D'Halluin K et al. (2008) Homologous recombination: a basis for targeted genome optimization in crop species such as maize. Plant Biotechnology Journal 6: 93-102

10. Nunez E et al. (2008) Nuclear receptor-enhanced transcription requires motorand LSD1-dependent gene networking in interchromatin granules. Cell 132: 996-1010 